\title{
Complex dynamics and its stabilization in an eco-epidemiological model with alternative food
}

\author{
Krishna Pada Das ${ }^{1}$
}

Received: 21 September 2016/Accepted: 27 September 2016/Published online: 6 October 2016

(C) Springer International Publishing Switzerland 2016

\begin{abstract}
The present paper deals with a predator-prey system with disease in the predator population and we have also studied the effect of alternative food on the system dynamics. We have analyzed the local stability of the biological feasible equilibria and introduced the concept of the ecological and the disease basic reproduction numbers. We have observed the community structure of our model system. We have performed extensive numerical simulation to observe the global behavior of our system. We have observed stable focus, limit cycle, period-doubling and chaos for variation of the force of infection. It is observed that when the infection level increases, the system enters into chaos from stable focus. We have also observed the role of alternative food on the chaotic dynamics. When the alternative food increases, the system remains chaotic dynamics but when the alternative food decreases, the system enters into a stable focus from chaos.
\end{abstract}

Keywords Disease in predator - Alternative food - Chaos . Period-doubling $\cdot$ Stable focus $\cdot$ Reproduction number

\section{Introduction}

Ecology and epidemiology are major fields of study in their own right but there are some common features between these two systems. The effect of disease in the ecological system is an important issue from mathematical and ecological point of view. In recent time many researchers are

Krishna Pada Das

krishnaisi@yahoo.co.in

1 Department of Mathematics, Mahadebananda Mahavidyalaya, P.O.-Barrackpore, Monirampore, India interested to study the ecological systems subject to epidemiological factors. Kermack and McKendrick (1927) represented a SIRS system in which the evolution of disease which got transmitted upon contact was described. Anderson and May (1986) were probably the first who considered the disease factor in the predator-prey system. A model for a disease spreading among the interacting populations was described by Hadeler and Freedman (1989). After pioneering work of Chattopadhyay and Arino (1999) on eco-epidemiology a number of studies have been performed in this direction, since transmissible disease in ecological situation can not ignored, see for instance (Freedman (1990); Venturino (2002); Xiao and Chen (2001).

Most of the previous studies such as Anderson and May (1986), Hadeler and Freedman (1989), Venturino (1994, (1995), Chattopadhyay and Arino (1999), Han et al. (2001), and Hethcote et al. (2004) mainly focused on parasitic infection in the prey species only, although some studies did consider infection in the predator through eating the prey (e.g. Anderson and May Anderson and May (1980); Hadeler and Freedman (1989); Venturino Venturino (1994)) or spread of disease in the predators (Venturino 2002). Venturino (2001) also studied the dynamics of two competing species when one of them is subject to a disease but the disease can not cross the species barrier. In recent times, Hsieh and Hsiao (2008) and Fenton and Rands (2006) considered a predator-prey system with disease in both populations. But the study of the dynamics of a predator prey system with an infected predator has great importance so long as the question of the predator control is concerned. To the best our knowledge, mathematical epidemiology is almost silent in this issue.

In the present study we consider a predator-prey system with disease in the predator species only. We pay attention 
to the chaotic dynamics and the role of alternative food on the chaotic dynamics. Though the study of chaos in an ecoepidemiological system is new but the literature of chaos in the ecological system is very rich. The terms chaos, strange attractor and fractal are becoming familiar to many, if not all, ecologists (Schaffer and Kot 1986a). The key feature of chaotic dynamics is the sensitive dependence on initial conditions that can lead to different results. An investigation by Gilpin (1979) showed that a system of one predator and two competing prey can exhibits chaotic behavior. Chaotic dynamics is common in a tri-trophic food chain and is of common interest to both modelers and experimental ecologists. Hastings and Powell (HP) (1991) produced a chaotic population system in a simple tri-trophic food chain model with type-II functional response. It is to be noted that the natural system seem to have no difficulty to switch from one state to another (chaos to order and order to chaos). After the work of Hastings and Powell (1991), many researchers stabilized the chaotic dynamic of their model by including various ecological factors (Rai and Sreenivasan 1993; Ruxton 1994). Schaffer and Kot (1985b) have been especially persuasive in their view that chaos may be a much more important phenomenon than ecologists had earlier believed. In particular, a number of simple ecological and epidemiological systems with seasonality in contact rates unequivocally demonstrate chaos (Schaffer and Kot 1986b). Schaffer and Kot (1985a) and Oslen et al. (1988) show that measles in New York, Baltimore and Denmark may be a specific example of this behavior. Recently Chatterjee et al. (2006) observed that the rate of infection and the predation rate are two prime factors that govern chaotic dynamic in the eco-epidemiological system.

In this study the alternative food is an important factor. Alternative food source for the predator population is an important factor in the interacting ecological species and inclusion of this factor in eco-epidemiological studies might give some interesting results. Predators do not generally feed on a single prey species, but will switch to alternative food sources when the density of the preferred prey is low (Murdoch 1969). It is argued that an alternative food source may play an important role in promoting the persistence of the predator-prey systems. It is also wellknown fact from foraging theory that when prey density drops below a threshold value due to infection in the preferred prey population or any other cause, optimally foraging predators will switch to alternative food sources, either by including the alternative food source in their diet (in a fine-grained environment) or by moving to the alternative food source (in a coarse-grained environment). Charnov (1976) published a well-known model of optimal diets that consists of a stepwise switch from a diet of profitable prey only to a mixed diet including alternative food. Fryxell and Lundberg (1994) have demonstrated using numerical simulation studies of one predator/twoprey models, that predator will switch to low-quality prey only when they have reduced the more profitable prey to low levels. They also observed that when prey density is low, such a switch will diminish predation pressure on the profitable prey. The presence of alternative food for a predator leads to reduction in equilibrium prey densities and is termed as apparent competition.

However, in the present study we consider a predator prey system with disease in the predator population and there is an alternative food source. We investigate the chaotic dynamics for variation of the force of infection and observe the role of alternative food on the chaotic dynamics. We have also introduced the concept of the ecological as well as the disease basic reproduction numbers. It can be defined as the expected number of off-spring a typical individual produces in its life or in epizootiology, as the expected number of secondary infections produced by a single infective individual in a completely susceptible population during its entire infectious period. While the concept of reproduction numbers was initially developed in demography already in the early 20th century Lotka (1925), they became a standard tool in epidemiology since the work of Anderson and May (1991) and Diekmann et al. (1990) . We will use reproduction numbers as helpful tools in determining the persistence or extinction of a species. This will alow us to categorize the community composition of prey, predators and disease. The threshold concept inherent in the reproduction numbers has been used in previous studies of eco-epidemiological models (Hadeler and Freedman 1989; Han et al. 2001; Xiao and Chen 2001; Hethcote et al. 2004).

The paper is organized as follows. In "Model formulation", we outline the mathematical model with some basic assumption. In "Model analysis" we study the stability of the equilibrium points and Hopf bifurcation and in "Numerical results" we have performed extensive numerical experiments. The article ends with a conclusion.

\section{Model formulation}

The Rosenzweig-MacArthurRosenzweig and MacArthur (1963) predator-prey model is given by

$$
\begin{aligned}
& \frac{d X}{d t}=R X\left(1-\frac{X}{K}\right)-\frac{A X P}{B+X} \\
& \frac{d P}{d t}=\frac{e A X P}{B+X}-D P
\end{aligned}
$$

where $X$ and $P$ are the prey and the predator populations. Prey population grow logistically with the carrying capacity $K$ and the intrinsic growth rate $R$. The predator 
consumes the prey according to the Holling type-II functional response. Here, $B$ represents the half saturation constant and $A$ is the searching efficiency constant for the predator. $e$ is the conversion efficiency and $D$ is the death rate of the predator.

To introduce a transmissible disease in the predator species of Rosenzweig-MacArthur (1) we make the following assumptions:

1. In absence of predator, the prey population grows logistically with carrying capacity $K \in R_{+}$and intrinsic birth rate $R \in R_{+}$.

2. We assume that disease spreads within the predator population so that total predator population $P=Y+Z$ can be split into the susceptible $(Y)$ and the infected (I) parts. The parasite is assumed to be transmitted. We further assume that the parasite attacks the predator population only. Xiao and Chen (2001) and many others have assumed a classical mass action incidence to describe the infection mechanism. It may be noted that if the degree of infectivity increases, many other mechanisms often come into the picture which tend to saturate the effect that a large numbers of parasites may have. Therefore, it is more reasonable to replace the simple mass action term by Holling type-II term in order to have a clear insight of the microparasite infection. Many studies in the epidemiological literature have considered the Holling type-II function to describe the infection mechanism.

With the above assumptions, the model (1) takes the following form:

$$
\begin{aligned}
& \frac{d X}{d t}=R X\left(1-\frac{X}{K}\right)-\frac{A X(Y+b Z)}{B_{1}+X} \\
& \frac{d Y}{d t}=\frac{A X\left(e_{1} Y+e_{2} b Z\right)}{B_{1}+X}-\frac{\lambda Y Z}{B_{2}+Y}-D_{1} Y \\
& \frac{d Z}{d t}=\frac{\lambda Y Z}{B_{2}+Y}-D_{2} Z
\end{aligned}
$$

System (2) has to be analyzed with the following initial conditions:

$X(0)>0, Y(0)>0, Z(0)>0$.

Here we describe the parameters. $R$ is the intrinsic growth rate of the prey population; $K$ is the carrying capacity of the prey population; $A$ is the predation rate of the both predator; $b$ is the predation efficiency of the infected predator; $B_{1}$ and $B_{2}$ are the half-saturation constants; $e_{1}$ and $e_{2}$ are the conversion factor of the susceptible and the infected predator respectively; $\lambda$ is the force of infection (transmission rate); $D_{1}$ and $D_{2}$ are the mortality rates of the susceptible and the infected predator respectively.

To reduce the number of parameters we adimensionalize the system (2) with the following scaling
$N=\frac{X}{K}, S=\frac{Y}{K}, I=\frac{Z}{K}, \quad$ and $\quad t=R T$.

Then system (2) takes the form,

$$
\begin{aligned}
& \frac{d N}{d t}=N(1-N)-\frac{a N(S+b I)}{1+b_{1} N} \\
& \frac{d S}{d t}=\frac{a N\left(e_{1} S+e_{2} b I\right)}{1+b_{1} N}-\frac{\beta S I}{1+b_{2} S}-d_{1} S \\
& \frac{d I}{d t}=\frac{\beta S I}{1+b_{2} S}-d_{2} I \\
& a=\frac{A K}{B_{1} R}, b_{1}=\frac{K}{B_{1}}, \beta=\frac{\lambda K}{B_{2} R}, b_{2}=\frac{K}{B_{2}}, \\
& d_{1}=\frac{D_{1}}{R}, d_{2}=\frac{D_{2}}{R}
\end{aligned}
$$

\section{Model analysis}

\section{Local stability of equilibrium points}

The system has four equilibrium points. The trivial equilibrium point $E_{0}(0,0,0)$ and the axial equilibrium point $E_{1}(1,0,0)$ exist for all parametric values. Disease free equilibrium point is $E_{2}(\bar{N}, \bar{S}, 0)$, where

$\bar{N}=\frac{d_{1}}{a e_{1}-b_{1} d_{1}}, \bar{S}=\frac{\left(a e_{1}-b_{1} d_{1}-d_{1}\right) e_{1}}{\left(a e_{1}-b_{1} d_{1}\right)^{2}}$.

The existence conditions of the disease free equilibrium point $E_{2}$ are

$a e_{1}-b_{1} d_{1}>0$ and $a e_{1}-b_{1} d_{1}-d_{1}>0$.

The interior equilibrium point is given by $E^{*}\left(N^{*}, S^{*}, I^{*}\right)$, where

$$
\begin{aligned}
S^{*} & =\frac{d_{2}}{\beta-b_{2} d_{2}}=E \\
I^{*} & =\frac{\left(\beta-b_{2} d_{2}\right)\left(1-N^{*}\right)\left(1+b_{1} N^{*}\right)-a d_{2}}{a b\left(\beta-b_{2} d_{2}\right)} \\
& =F\left(1-N^{*}\right)\left(1+b_{1} N^{*}\right)-G
\end{aligned}
$$

where $F=\frac{1}{a b}$ and $G=\frac{a d_{2}}{a b\left(\beta-b_{2} d_{2}\right)}$.

$N^{*}$ is the positive root of the equation

$Q_{1} N^{3}+Q_{2} N^{2}+Q_{3} N+Q_{4}=0$

where

$$
\begin{aligned}
& Q_{1}=b_{1} F L \\
& Q_{2}=F L\left(1-b_{1}\right)-\beta E F b_{1} \\
& Q_{3}=L(G-F)-\beta E F\left(1-b_{1}\right)-E\left(1+b_{2} E\right)\left(a e_{1}-b_{1} d_{1}\right) \\
& Q_{4}=\beta E F+d_{1} E\left(1+b_{2} E\right)-\beta E G
\end{aligned}
$$


Here $L=a e_{2} b\left(1+b_{2} E\right)-\beta b_{1} E$.

The Jacobian matrix $J$ of the system (3) at any arbitrary point $(N, S, I)$ is given by $J=\left(j_{i j}\right)_{3 \times 3}$

where

$$
\begin{aligned}
& j_{11}=1-2 N-\frac{a(S+b I)}{\left(1+b_{1} N\right)^{2}}, \\
& j_{12}=\frac{-a N}{1+b_{1} N}, \quad j_{13}=\frac{-a b N}{1+b_{1} N} \\
& j_{21}=\frac{a\left(e_{1} S+e_{2} b I\right)}{\left(1+b_{1} N\right)^{2}}, \quad j_{22}=\frac{e_{1} a N}{1+b_{1} N}-\frac{\beta I}{\left(1+b_{2} S\right)^{2}}-d_{1}, \\
& j_{23}=\frac{\left.a N e_{2} b\right)}{1+b_{1} N}-\frac{\beta S}{1+b_{2} S} \\
& j_{31}=0, \quad j_{32}=\frac{\beta I}{\left(1+b_{2} S\right)^{2}}, \quad j_{33}=\frac{\beta S}{1+b_{2} S}-d_{2}
\end{aligned}
$$

Theorem 1 The trivial equilibrium point $E_{0}$ is always unstable. The axial equilibrium point $E_{1}$ is locally stable if $R_{01}<1$ where $R_{01}=\frac{1}{d_{1}}\left(\frac{e_{1} a}{1+b_{1}}\right)$. The disease free equilibrium point $E_{2}$ is locally stable if $R_{02}<1$ and $\frac{a b \bar{S}}{\left(1+b_{1} \bar{N}\right)^{2}}<1$, where $R_{02}=\frac{1}{d_{2}}\left(\frac{\beta \bar{S}}{1+b_{2} \bar{S}}\right)$

Proof Since an eigenvalue associated with the Jacobian matrix at $E_{0}$ is 1 , so $E_{0}$ is an unstable equilibrium point.

From the Jacobian matrix computed around $E_{1}$ it is observed that eigenvalues of matrix are $-1, \frac{a e_{1}}{1+b_{1}}-d_{1}, d_{2}$. So, the equilibrium point $E_{1}$ is stable if $\frac{a e_{1}}{1+b_{1}}-d_{1}<0$ which implies $R_{01}<1$.

The Jacobian matrix at $E_{2}$ is given by

$$
J_{2}=\left[\begin{array}{ccc}
-\bar{N}+\frac{a b \bar{N} \bar{S}}{\left(1+b_{1} \bar{N}\right)^{2}} & -\frac{a \bar{N}}{1+b_{1} \bar{N}} & -\frac{a b \bar{N}}{1+b_{1} \bar{N}} \\
\frac{e_{1} a \bar{S}}{\left(1+b_{1} \bar{N}\right)^{2}} & 0 & -\frac{\beta \bar{S}}{1+b_{2} \bar{S}} \\
0 & 0 & \frac{\beta \bar{S}}{1+b_{2} \bar{S}}-d_{2}
\end{array}\right]
$$

The characteristic roots of the Jacobian matrix $J_{2}$ are $\frac{\beta \bar{S}}{1+b_{2} S}-d_{2}$ and roots of the equation

$\rho^{2}+\left(\bar{N}-\frac{a b \bar{N} \bar{S}}{\left(1+b_{1} \bar{N}\right)^{2}}\right) \rho+\frac{a^{2} e_{1} \bar{S} \bar{N}}{\left(1+b_{1} \bar{N}\right)^{3}}=0$

Hence $E_{2}$ is stable if $\frac{\beta \bar{S}}{1+b_{2} \bar{S}}-d_{2}<0$ and $\bar{N}-\frac{a b \bar{N} \bar{S}}{\left(1+b_{1} \bar{N}\right)^{2}}>0$ which implies the conditions $R_{02}<1$ and $\frac{a b \bar{S}}{\left(1+b_{1} \bar{N}\right)^{2}}<1$.

Theorem 2 The interior point $E^{*}\left(N^{*}, S^{*}, I^{*}\right)$ for the system (3) is locally asymptotically stable if the following conditions are hold: (i) $\frac{a b_{1}(S+b I)}{\left(1+b_{1} N\right)^{2}}<1$

(ii) $\frac{\beta b_{2} S}{\left(1+b_{2} S\right)^{2}}<\frac{a e_{2} b}{S\left(1+b_{1} N\right)}$

(iii) $A_{32} A_{22}+A_{13} A_{21}>0$

Proof The Jacobian matrix at the interior point $E^{*}\left(N^{*}, S^{*}, I^{*}\right)$ is $V=\left(A_{i j}\right)_{3 \times 3}$ where

$$
\begin{aligned}
& A_{11}=-N+\frac{a b_{1} N(S+b I)}{\left(1+b_{1} N\right)^{2}}, \quad A_{12}=\frac{-a N}{1+b_{1} N}, A_{13}=\frac{-a b N}{1+b_{1} N} \\
& A_{21}=\frac{a\left(e_{1} S+e_{2} b I\right)}{\left(1+b_{1} N\right)^{2}}, \quad A_{22}=\frac{\beta b_{2} I S}{\left(1+b_{2} S\right)^{2}}-\frac{a N e_{2} b I}{S\left(1+b_{1} N\right)}, \\
& A_{23}=\frac{a N e_{2} b}{1+b_{1} N}-\frac{\beta S}{1+b_{2} S} \\
& A_{31}=0, A_{32}=\frac{\beta I}{\left(1+b_{2} S\right)^{2}}, A_{33}=0 .
\end{aligned}
$$

The characteristic equation of Jacobian matrix is given by $\rho^{3}+\sigma_{1} \rho^{2}+\sigma_{2} \rho+\sigma_{3}=0$

where

$$
\begin{aligned}
& \sigma_{1}=-\left(A_{11}+A_{22}\right) \\
& \sigma_{2}=A_{11} A_{22}-A_{23} A_{32}-A_{12} A_{21} \\
& \sigma_{3}=A_{23} A_{32} A_{11}-A_{13} A_{21} A_{32} \\
& \sigma_{1} \sigma_{2}-\sigma_{3}=-\left(A_{11}+A_{22}\right)\left[A_{11} A_{22}-A_{12} A_{21}\right] \\
& +A_{23}\left(A_{32} A_{22}+A_{13} A_{21}\right)
\end{aligned}
$$

If $A_{11}<0, A_{22}<0$ and $A_{32} A_{22}+A_{13} A_{21}>0$, then $\sigma_{1}>0$, $\sigma_{3}>0$ and $\sigma_{1} \sigma_{2}-\sigma_{3}>0$.

Using the Routh-Hurwitz criteria we observe that the system (3) is stable around the positive equilibrium point $E^{*}$ if the conditions stated in the theorem hold.

\section{Community composition of model system: ecological and disease basic reproduction numbers}

Here we analyze the community structure of the model system. It can be completely explained by two threshold quantities that the ecological and the disease basic reproduction numbers. Note that these numbers can be obtained independently by the stability analysis (see Theorem 1). We will also explain the biological significance of the ecological as well as the disease basic reproduction numbers.

We define the reproduction number $R_{01}$ by

$R_{01}=\frac{1}{d_{1}}\left(\frac{e_{1} a}{1+b_{1}}\right)$

which determines the local stability of the axial equilibrium $E_{1}$. Here $\frac{e_{1} a}{1+b_{1}}$ is the birth rate of the susceptible predator at 
$E_{1}$ and $\frac{1}{d_{1}}$ is the mean lifespan of a predator. Subsequently, their product gives the mean number of newborn susceptible predators by a predator, which can be interpreted as the "ecological" basic reproduction number. We also note that this term, first formulated and explained by Pielou (1969), is the average number of the prey converted to the predator biomass in a course of the predator's life span Hethcote et al. (2004). $R_{01}<1$ implies the predators will become extinct and only the prey species survive in the system.

Another important reproduction number $R_{02}$ is defined by

$R_{02}=\frac{1}{d_{2}}\left(\frac{\beta \bar{S}}{1+b_{2} \bar{S}}\right)$

which determines the local stability of the disease free equilibrium point $E_{2}$. Here $\frac{\beta \bar{S}}{1+b_{2} S}$ is the infection rate of a new infective prey appearing in a totally the susceptible prey population and $\frac{1}{d_{2}}$ is the duration of infectivity of an infective prey, the product of which is the disease basic reproduction. $R_{02}<1$ implies the infected predator will be washed out and the prey and the susceptible predator survive in the system.

The entire community composition i.e. persistence of (i) the prey alone (ii) the prey and the susceptible prey can be predicted by biologically meaningful reproduction numbers. The prey can always grows and survives. Hence the stationary states $E_{0}$ is always unstable.

\section{Hopf bifurcation}

Theorem 3 The rate of infection $\beta$ crosses a critical value $\beta^{*}$, the system enters into Hopf-bifurcation around the positive equilibrium $E^{*}$ if the following conditions hold:

(i) $\sigma_{1}\left(\beta^{*}\right)>0$

(ii) $\sigma_{1}\left(\beta^{*}\right) \sigma_{2}\left(\beta^{*}\right)-\sigma_{3}\left(\beta^{*}\right)=0$

(iii) $\left[\sigma_{1}\left(\beta^{*}\right) \sigma_{2}\left(\beta^{*}\right)\right]^{\prime}<\sigma_{3}^{\prime}\left(\beta^{*}\right)$

Proof We assume that the steady state $E^{*}$ is asymptotically stable, we would like to know if $E^{*}$ will lose its stability when one of the parameters changes. We choose $\beta$, the force of infection as the bifurcation parameter, we can see that if there exists a critical value $\beta^{*}$ such that

$\sigma_{1}\left(\beta^{*}\right)>0$,

$\sigma_{1}\left(\beta^{*}\right) \sigma_{2}\left(\beta^{*}\right)-\sigma_{3}\left(\beta^{*}\right)=0,\left[\sigma_{1}\left(\beta^{*}\right) \sigma_{2}\left(\beta^{*}\right)\right]^{\prime}<\sigma_{3}^{\prime}\left(\beta^{*}\right)$.

For the Hopf-bifurcation to occur at $\beta=\beta^{*}$, the characteristic equation must be of the form,

$\left(\rho^{2}\left(\beta^{*}\right)+\sigma_{2}\left(\beta^{*}\right)\right)\left(\rho\left(\beta^{*}\right)+\sigma_{1}\left(\beta^{*}\right)\right)=0$,

which has three roots $\rho_{1}\left(\beta^{*}\right)=i \sqrt{ } \sigma_{2}\left(\beta^{*}\right), \rho_{2}=-i \sqrt{ } \sigma_{2}\left(\beta^{*}\right)$, $\rho_{3}=-\sigma_{1}\left(\beta^{*}\right)<0$.
To see if Hopf bifurcation occurs at $\beta=\beta^{*}$, we need to verify the transversality condition

$\left[\frac{d \operatorname{Re}(\rho(\beta))}{d \beta}\right]_{\beta=\beta^{*}} \neq 0$.

For all $\beta$, the roots are in general of the form

$\rho_{1}(\beta)=\mu(\beta)+i v(\beta)$,

$\rho_{2}(\beta)=\mu(\beta)-i v(\beta)$,

$\rho_{3}(\beta)=-\sigma_{1}(\beta)$

Now, we shall verify the transversality condition

$\frac{d \operatorname{Re}\left(\rho_{j}(\beta)\right)}{d \beta} \neq 0, j=1,2$.

Substituting $\rho_{j}(\beta)=\mu(\beta) \pm i v(\beta)$, into (5) and calculating the derivative, we have

$$
\begin{aligned}
& K(\beta) \mu^{\prime}(\beta)-L(\beta) v^{\prime}(\beta)+M(\beta)=0 \\
& K(\beta) \mu^{\prime}(\beta)+L(\beta) v^{\prime}(\beta)+N(\beta)=0
\end{aligned}
$$

where

$$
\begin{aligned}
& K(\beta)=3 \mu^{2}(\beta)+2 \sigma_{1}(\beta) \mu(\beta)+\sigma_{2}(\beta)-3 v^{2}(\beta) \\
& L(\beta)=6 \mu(\beta) v(\beta)+2 \sigma_{1}(\beta) v(\beta) \\
& M(\beta)=\mu^{2}(\beta) \sigma_{1}^{\prime}(\beta)+\sigma_{2}^{\prime}(\beta) \mu(\beta)+\sigma_{3}^{\prime}(\beta)-\sigma_{1}^{\prime}(\beta) v^{2}(\beta) \\
& N(\beta)=2 \mu(\beta) v(\beta) \sigma_{1}^{\prime}(\beta)+\sigma_{2}^{\prime}(\beta) v(\beta)
\end{aligned}
$$

Notice that $\mu\left(\beta^{*}\right)=0, v\left(\beta^{*}\right)=\sqrt{ } \sigma_{2}\left(\beta^{*}\right)$, we have

$$
\begin{aligned}
& K(\beta)=-2 \sigma_{2}\left(\beta^{*}\right), L\left(\beta^{*}\right)=2 \sigma_{1}\left(\beta^{*}\right) \sqrt{ } \sigma_{2}\left(\beta^{*}\right) \\
& M\left(\beta^{*}\right)=\sigma_{3}^{\prime}\left(\beta^{*}\right)-\sigma_{1}^{\prime}\left(\beta^{*}\right) \sigma_{2}\left(\beta^{*}\right), N\left(\beta^{*}\right)=\sigma_{2}^{\prime}\left(\beta^{*}\right) \sqrt{ } \sigma_{2}\left(\beta^{*}\right)
\end{aligned}
$$

Solving for $\mu^{\prime}\left(\beta^{*}\right)$ from system (7) we have

$$
\begin{aligned}
{\left[\frac{d \operatorname{Re}\left(\rho_{j}(\beta)\right)}{d \beta}\right]_{\beta=\beta^{*}} } & =\mu^{\prime}(\beta)_{\beta=\beta^{*}}=-\frac{L\left(\beta^{*}\right) N\left(\beta^{*}\right)+K\left(\beta^{*}\right) M\left(\beta^{*}\right)}{K^{2}\left(\beta^{*}\right)+L^{2}\left(\beta^{*}\right)} \\
& =\frac{\sigma_{3}^{\prime}\left(\beta^{*}\right)-\sigma_{1}^{\prime}\left(\beta^{*}\right) \sigma_{2}\left(\beta^{*}\right)-\sigma_{1}\left(\beta^{*}\right) \sigma_{2}^{\prime}\left(\beta^{*}\right)}{\sigma_{1}^{2}\left(\beta^{*}\right)+\sigma_{2}\left(\beta^{*}\right)}>0
\end{aligned}
$$

if $\left[\sigma_{1}\left(\beta^{*}\right) \sigma_{2}\left(\beta^{*}\right)\right]^{\prime}<\sigma_{3}^{\prime}\left(\beta^{*}\right)$ and

$\rho_{3}\left(\beta^{*}\right)=-\sigma_{1}\left(\beta^{*}\right)<0$

Thus the transversality conditions hold and hence Hopf bifurcation occurs at $\beta=\beta^{*}$.

Hence the theorem.

Remark If there exists a critical value of force infection $\beta^{*}$ such that $\sigma_{1}\left(\beta^{*}\right)>0, \sigma_{1}\left(\beta^{*}\right) \sigma_{2}\left(\beta^{*}\right)-\sigma_{3}\left(\beta^{*}\right)=0$ and $\left[\sigma_{1}\left(\beta^{*}\right) \sigma_{2}\left(\beta^{*}\right)\right]^{\prime}<\sigma_{3}^{\prime}\left(\beta^{*}\right)$, then when $\beta>\beta^{*}$, the steady state $E^{*}$ is stable; when $\beta=\beta^{*}, E^{*}$ loses its stability and the Hopf bifurcation occurs at $E^{*}$, when $\beta<\beta^{*}, E^{*}$ 
becomes unstable and a family of periodic solutions bifurcates from $E^{*}$.

\section{Numerical results}

To observe the global dynamics of our system we perform the extensive numerical simulations. Here two key parameters viz the force of infection $(\beta)$ and the alternative food source of the predator i.e. the carrying capacity $(k)$. First we will observe dynamical behavior of the model system for variation of the force of infection in the predator population and finally observe the role of alternative food. We consider a set of parameter values (most of the values are taken from Hastings and Powell 1991) $a_{1}=4.8, \beta=0.074, b_{1}=3.0, b_{2}=2.0, d_{1}=0.4$, $d_{2}=0.01, e=0.02, b=0.001$. For these set of parameter values we observe that the stability conditions of the interior equilibrium point are satisfied and all species coexist in stable distribution (Fig. 1). Now we increase the values of $\beta$ from 0.074 to 0.078 and observe that the stability conditions are not satisfied and the stable distribution of all species become unstable through limit cycle oscillations (Fig. 2). Now we will explain the reason of oscillations in our model system. Whenever we increase the force of infection in the predator population, the susceptible predator decreases and the infected predator increases due to newly recruited the infected predator and the predation pressure on the prey population increases. As a results it is found that the biomass level of the prey and the susceptible predator decreases and the biomass level of the infected predator increases and such type of mechanism produces limit cycle oscillations. So it is clear from Fig. 2 that the disease introduction in the predator population generates limit cycle oscillations of all species. Existing mathematical models suggest that disease introduction into the predator population tends to destabilize established predatorprey communities. This has been observed for microparasites with both direct (Anderson and May 1986) and indirect life cycles (Dobson 1988; Fenton and Rands 2006). Macroparasitic models generally have a tendency to unstable dynamics, because they consider the parasite burden in the host in an additional equation (Anderson and May 1980).

If we further increase the values of the force of infection of the predator population, we observe that limit cycle oscillations becomes period-doubling (Fig. 3). It is also noticed that if we increase the values of $\beta$ from 0.082 to 0.1 , period-doubling becomes irregular oscillations and period of these oscillations can not be counted and such type of irregular oscillations is called chaos (Fig. 4). So it is clear that when the force of infection increase, the limit cycles oscillations enter into period-doubling and finally period-doubling enters into chaos. In this context, the term chaos can be defined as bounded aperiodic fluctuations with sensitive dependence on initial conditions. Under chaotic conditions, the population abundances never show a precisely repeated pattern over time; such patterns are only observable in the populations at equilibrium or at stable limit cycles. Theoreticians can clearly define parameter ranges of mathematical models that create chaotic behavior in idealized biological systems . To get a clear region of chaotic dynamics we draw a bifurcation
Fig. 1 Figure depicts the stable distribution of all three species for $a_{1}=4.8, \beta=$ $0.074, b_{1}=3.0, b_{2}=$ $2.0, d_{1}=0.4, d_{2}=0.01$, $e=0.02, b=0.001$, $r=0.0, k=0.0$
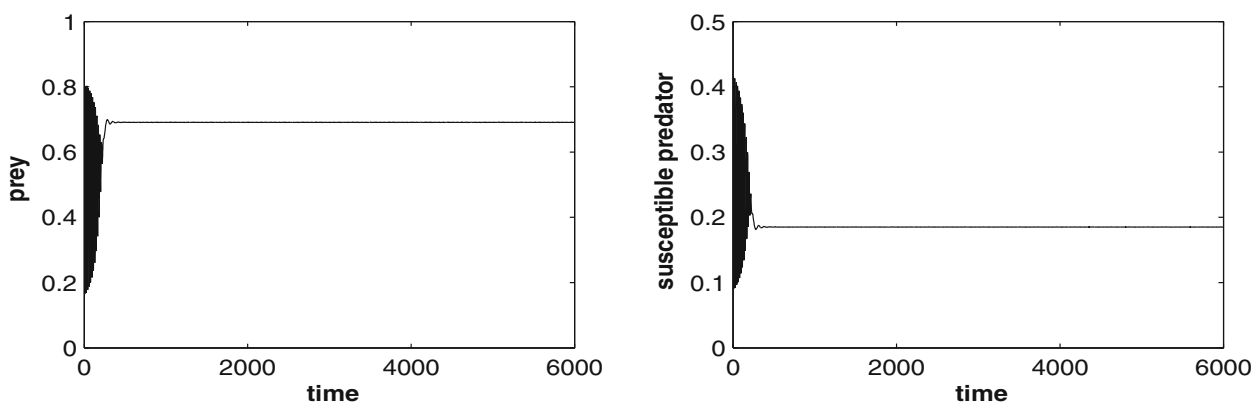

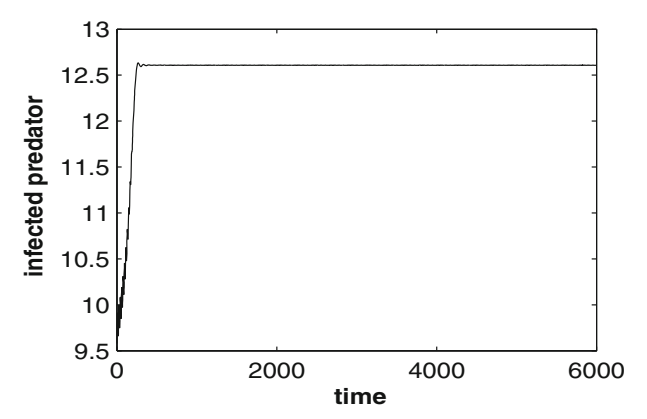


Fig. 2 Figure depicts the limit cycle oscillation for $\beta=0.078$ and other parameters fixed as given in Fig. (1)
Fig. 3 Figure depicts perioddoubling for $\beta=0.082$ and other parameters fixed as given in Fig. (1)
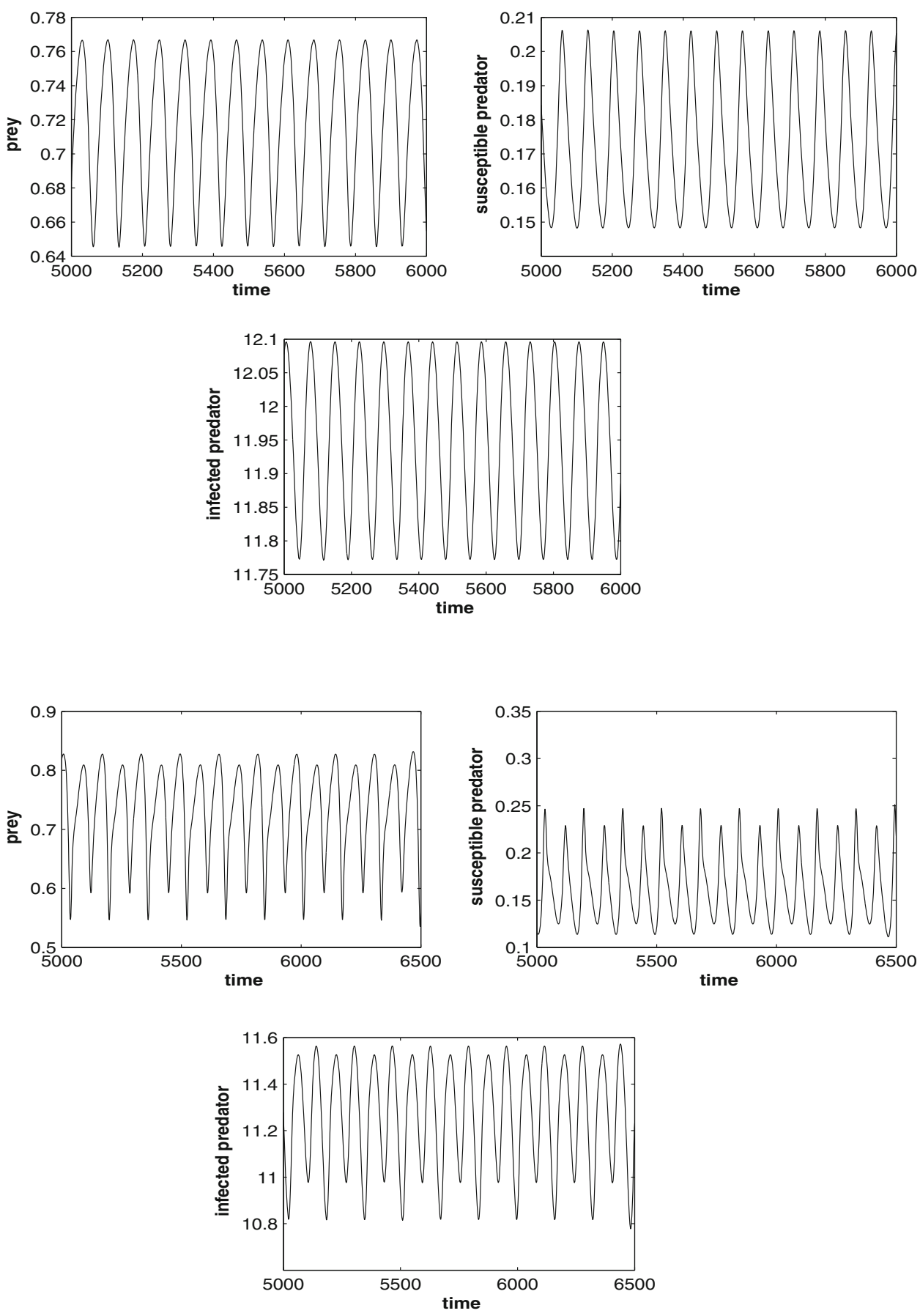

diagram (Fig. 5) for $\beta$ and we found that the system shows stable dynamics for $0.074 \leq 0.078$; limit cycles for $0.078 \leq 0.082$, period-doubling for $0.082 \leq 0.1$ and chaos for $\beta \geq 0.1$.

Now we will observe the role of alternative food on the chaotic dynamics. For this we introduce alternative food source in the system (3). We introduce a logistic growth law in the susceptible predator's equation. This is because that the predator is a generalist with a resource other than the prey available to it (i.e. humans, leopards and dogs). similar models are discussed by van Baalen et al. (2001). This is in contrast to other predator-prey models which assume that the predator is a specialist that is completely dependent on the prey. Almost all predators will attempt to switch to another prey when the preferred prey is in low numbers and they may also resort to scavenging or a herbivorous diet if possible Murdoch (1969); Van Baalen et al. (2001). An example of a predator-prey system with 
Fig. 4 Figure depicts the chaotic dynamics for $\beta=0.1$ and other parameters fixed as given in Fig. (1)
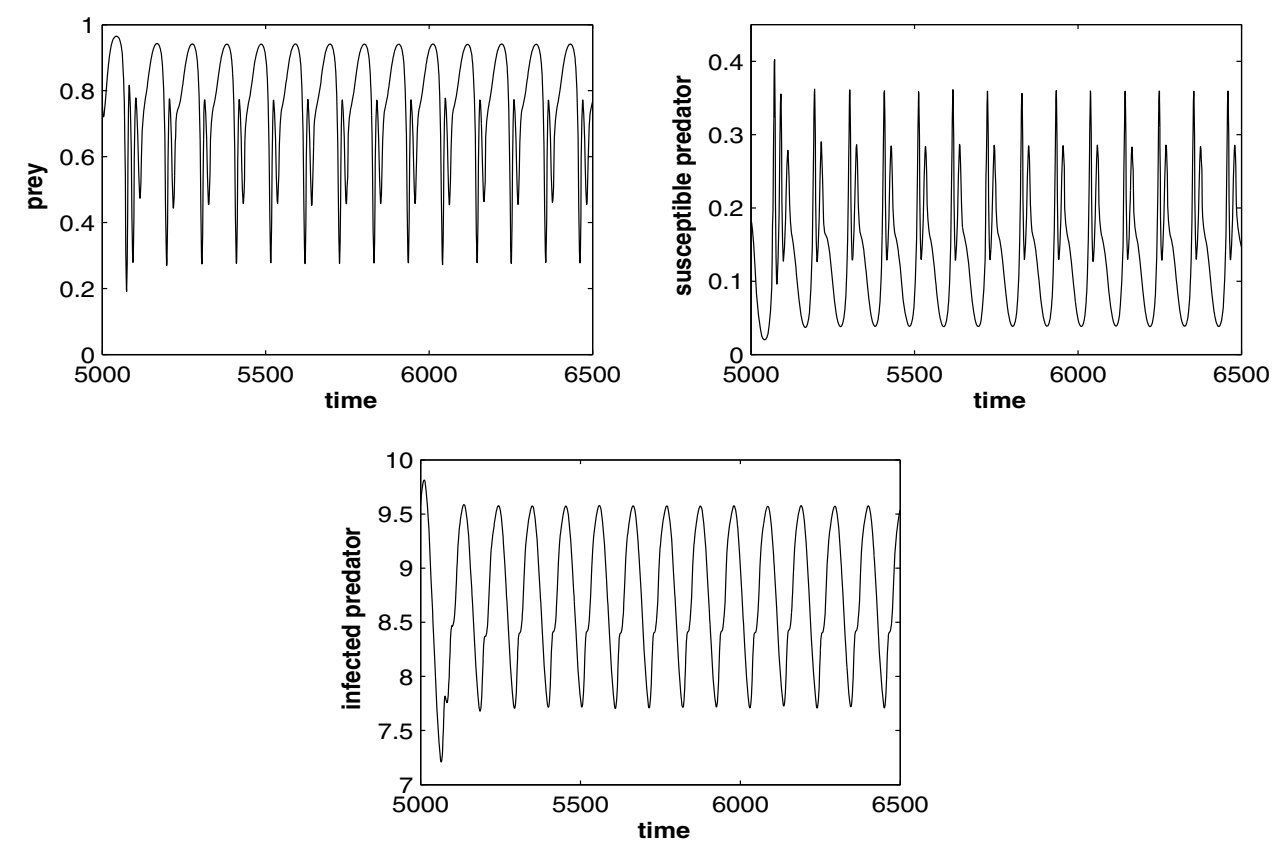

Fig. 5 Figure shows limit cycle, period-doubling, chaos for $\beta \in[0.075,0.1]$ and other parameters fixed as given in Fig. (1)
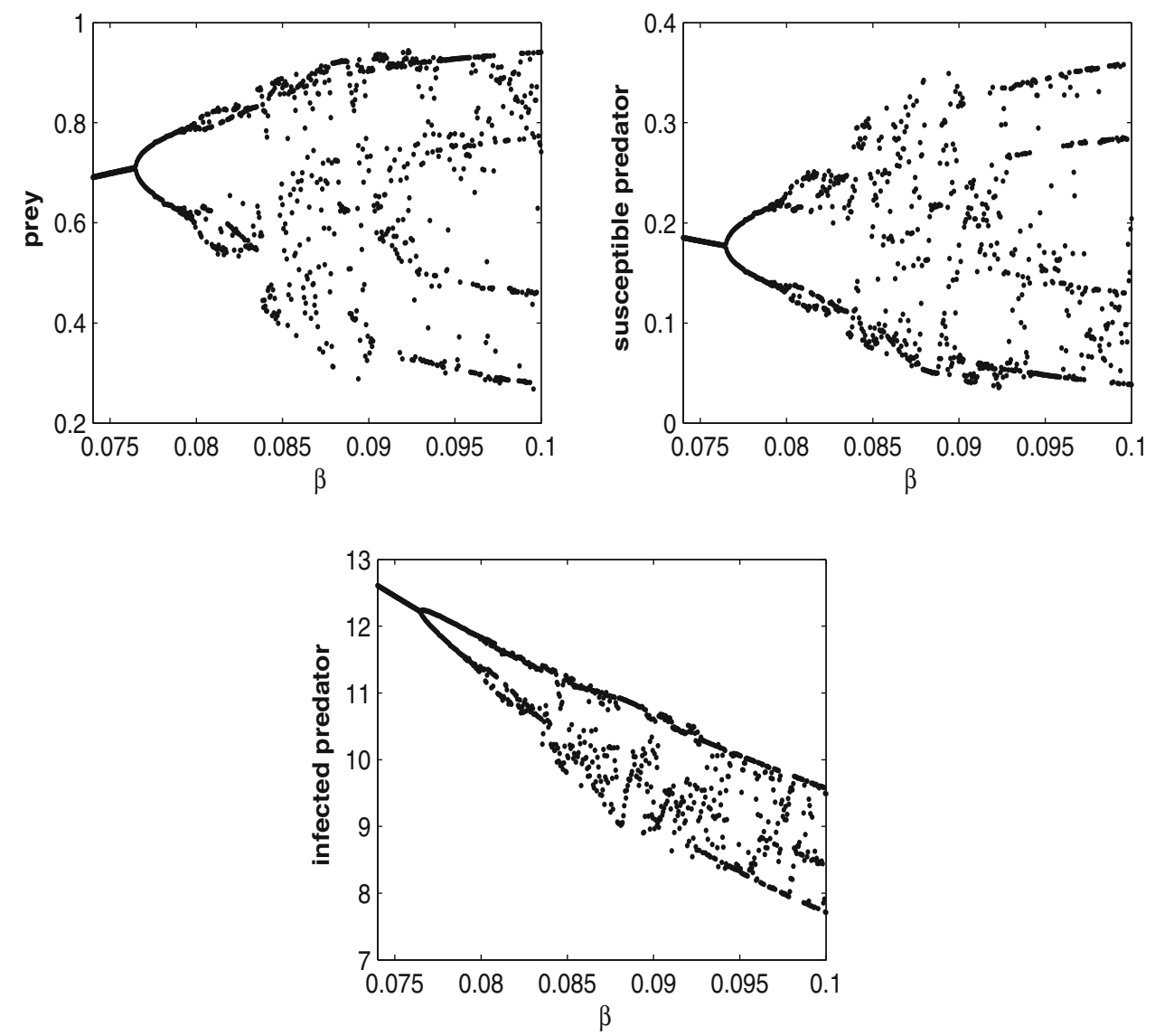

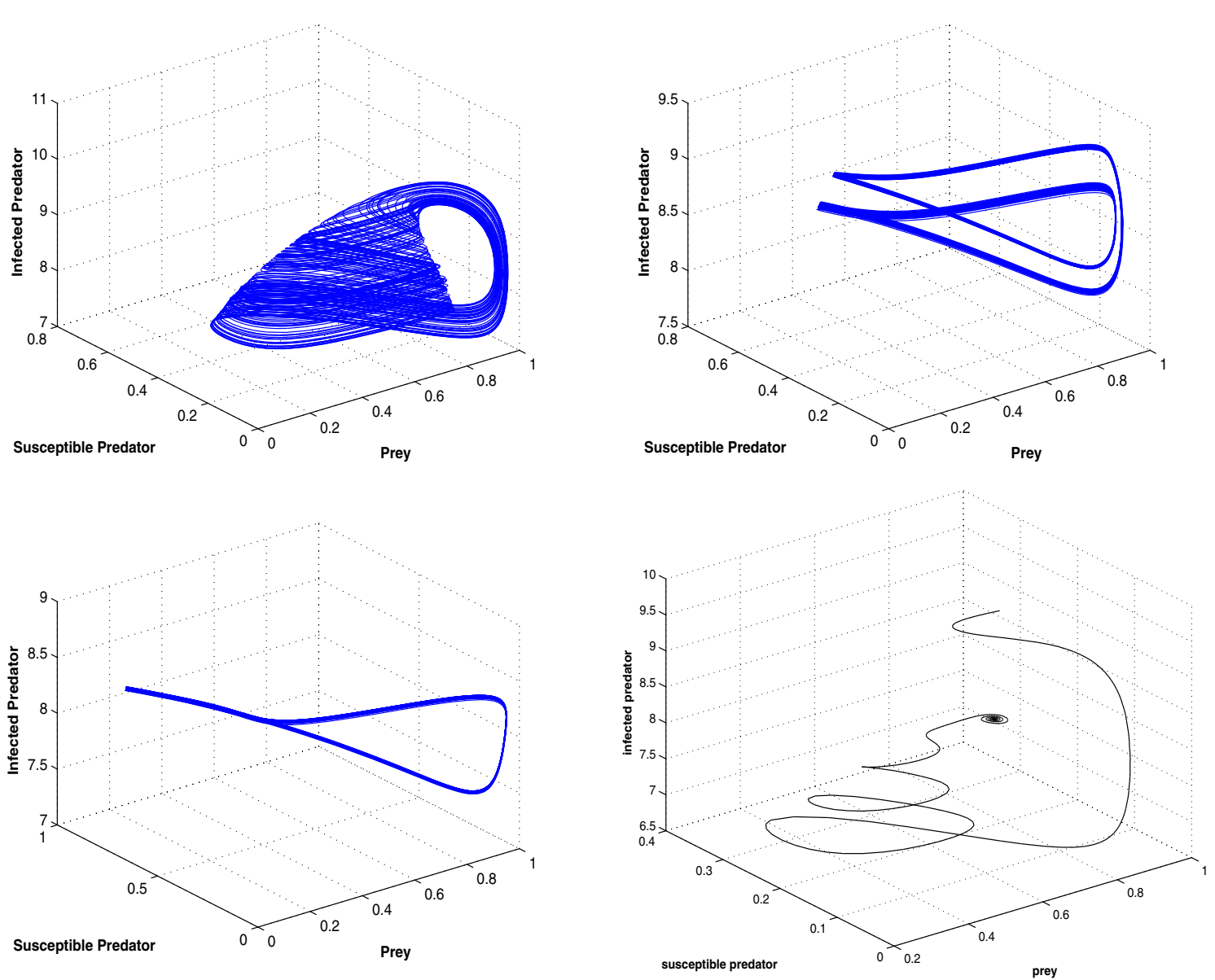

Fig. 6 a The strange chaotic attractor for $k=0.3, r=0.02$, b period-doubling for $k=0.12, r=0.02$, $\mathbf{c}$ depicts limit cycle oscillation for $k=0.08, r=0.02$, $\mathbf{d}$ stable focus for $k=0.04, r=0.02$ and all other parameters fixed as given in Fig. (4)

alternative food source is the hare-caribou-lynx relationship in Newfoundland. Now we introduce logistic growth in the predator population and the model system can be written as

$$
\begin{aligned}
& \frac{d N}{d t}=N(1-N)-\frac{a N(S+b I)}{1+b_{1} N} \\
& \frac{d S}{d t}=S\left(r-d_{1}-\frac{S}{k}\right)+\frac{a N\left(e_{1} S+e_{2} b I\right)}{1+b_{1} N}-\frac{\beta S I}{1+b_{2} S} \\
& \frac{d I}{d t}=\frac{\beta S I}{1+b_{2} S}-d_{2} I
\end{aligned}
$$

where $r$ is growth rate for alternative food and $k$ is the carrying capacity.

It is clear from above discussion that when the force of infection increases, the system enters into chaotic dynamics. We will observe the role of alternative food on the occurrence and control of chaotic dynamics. From Fig. 6 it is observe that the system shows chaotic dynamics for $r=0.02, k=0.3$; period-doubling for $k=0.12$; limit cycle oscillations for $k=0.08$ and finally the system enters into stable focus for $k=0.04$. So it is clear that if we decreases the source of alternative food, the system settles down into stable focus through from chaos to period-doubling; period-doubling to limit cycle; limit cycle to stable focus. If we increase the source of alternative food, the system remains in chaotic position. We observe from this diagram that when the level of alternative food is high the system shows chaotic dynamics and when the level of alternative food is low, the system shows stable dynamics. If we decrease the values of $k$ i.e. the source of alternative food, the biomass level of predator population decreases and as a result it is observed that the predation pressure on prey population decreases. When the predation pressure on the prey population decreases the biomass level of the prey population increases. Such type coupling interaction helps chaotic dynamics to reduce period-doubling; period-doubling to limit cycle and limit cycle to stable focus.

Finally our numerical results lead to a conclusion that when infection level is high system shows chaotic behavior and when infection level is low system shows stable focus. It is also observe that in higher level of infection, if source of alternative food increases the chaotic dynamics remains 
in the system but if the source of alternative food decreases chaotic dynamics converges to stable focus. Our findings are also of relevance for biological control as alternative food can be used as control agents of undesirable species such as biological invaders. This study interestingly suggests that the alternative food can have regulating effects on more than one trophic level and be utilized for management purposes in multi species systems. This study provides insightful the ecological and the disease reproduction numbers for understanding how disease propagation in predator population in the presence of alternative food structure community composition.

Now we will compare our study with the study of Mandal et al. (2009). Mandal et al. (2009) consider a predator-prey system with disease in the prey population. They observe the dynamics of such a system under the influence of severe as well as unnoticeable parasite attack and also the alternative food sources for the predator population. They assume the predator population will prefer only the infected prey for their diet as those are more vulnerable. Their results indicate that in the case of severe parasitic attack the predator population will prefer the alternative food source and not the infected one. But the strategy is reverse in the case of unnoticeable parasite attack. In our proposed system we have considered a predator-prey model with disease circulating in the predator population only and there is a alternative food source. We have observed that when infection level is high the system shows chaotic dynamics and when the infection level is low the system shows stable focus. We also observe that in higher infection level if the source of alternative food increases the chaotic dynamics remains same but if the source of alternative food decreases the chaotic dynamics reduces to stable focus.

We also present a comparative study with most of the earlier studies. Hadeler and Freedman (1989) considered a predator-prey model where both species are subjected to parasitism, is developed and analyzed. They also assumed that the predators could get infection by eating prey and the prey could obtained the disease from parasites spread into the environment by predators. They obtained a threshold condition above which an endemic equilibrium or an endemic periodic solution could arise in the case where there was coexistence of the predator with the uninfected prey. Furthermore, they also showed that in the case where the predator can not survive only on the prey in a diseasefree environment, the parasitization could lead to persistence of the predator since the predator could only survive on the predator if some of the prey were more easily captured due to being diseased, provided a certain threshold for disease transmission is surpassed. Hethcote et al.Hethcote et al. (2004) proposed a predator-prey model with logistic growth in prey to include an SIS infection with standard incidence in the prey population with the infected prey being more vulnerable to predation. They discovered several interesting cases where the disease infection in the prey could promote coexistence. In the work of Hsieh and Hsiao (2008), the predator can be infected upon contact with or being in the those vicinity of an infected prey during the process of predation, but the predator can not infect each other. Hsieh and Hsiao (2008) observed that infected predator plays a minor and indirect role in the spread of disease mainly due to the assumption that the infected predators are unable to infect other members of the population. Recently Hilker and Schmitz (2008) considered the invasion of a resident predator-prey system by an infectious disease with frequency-dependent transmission spreading within the predator population. They had derived biologically plausible and insightful quantities (demographic and epizootiolgical reproduction numbers) that allow them to completely determine community composition. Their findings contradict predictions from previous models suggesting a destabilizing effect of parasites and they show that predator infection counteracts the paradox of enrichment. In the present study we consider a predator-prey model with disease circulating in the predator population and there is an alternative food source. In recent time Chatterjee et al. (2006) proposed and analyzed an eco-epidemiological model to observed the occurrence and control of chaos. They concluded that along with the rate of infection, the rate of predation also plays a pivotal role for monitoring the dynamics of the system. Upadhyay et al. (2007) observed chaotic dynamics in ecoepidemiological model when some key parameters attain their critical values. They have tried to explain the unusual deaths of fish and fish eating birds in the Salton Sea using the simulation results and they have also suggested some possible measures to avoid chaos in such natural systems. Chatterjee et al. (2007) considered the model proposed by Chatterjee et al. (2006) and modified the model by assuming that the disease transmission follows standard incidence law. They compared the dynamical nature of the two systems numerically for a wider range of force of infection. Their observations indicated that the phenomenon of rarity or non-occurrence of chaos in their proposed model is well defined if the mode of disease transmission follows standard incidence. Das et al. (2009) modified Hasting and Powell's (HP) (1991) model by introducing disease in the prey population and observed that disease in the prey population as well as body size of the intermediate predator are the key parameters for controlling the chaotic dynamics observed in original HP model. Das et al. (2010) considered an eco-epidemiological model with disease-induced predator mortality function and observed that disease-induced mortality of the predator due to ingested infected prey may prevent the occurrence 
of chaos. Stiefs et al. (2009) have studied an eco-epidemic model with two trophic levels in which the dynamics is determined by predator-prey interactions as well as the vulnerability of the predator to a disease. Using the concept of generalized models they showed that for certain classes of eco-epidemic models quasi-periodic and chaotic dynamics is generic and likely to occur. We pay attention to the chaotic dynamics for variation of force of infection. We observe role of alternative food on the chaotic dynamics. We also introduce the concept of ecological as well as disease basic reproduction number and analyze the community structure of our model system.

\section{Conclusion}

Infectious diseases can have regulating effects not only on their host population, but also on their species their host interacts with Anderson and May (1986). Ecologists and epizootiologists alike become increasingly interested in the structuring the effects of parasites and pathogens within food webs and multiple-species communities (Dobson and Hudson 1986; Sait et al. 2000; Holt 2003). In this paper we have considered a predator-prey model with disease circulating in the predator population only and we have also considered an alternative food source of the predator population. We have analyzed the local stability of the system around the different biological feasible equilibria and introduced the ecological as well as the disease basic reproduction numbers. We have analyzed the community structure of our model system by the help of these numbers. To study the global dynamics we have performed extensive numerical simulation. We have observed that system enters into chaotic dynamics from stable focus for increasing the infection rate. In higher infection rate we have observed the role of alternative food in our proposed system. In higher infection rate if the alternative food increases the chaotic dynamics remain same but if the alternative food decreases the chaotic dynamics reduces to stable focus.

\section{References}

Anderson RM, May RM (1980) Infectious diseases and population cycles of forest insects. Science 210:658-661

Anderson RM, May RM (1986) The invasion, persistence and spread of infectious diseases within animal and plant communities. Philos Trans R Soc Lond B 314:533-570

Anderson RM, May RM (1991) Infectious diseases of humans. Dynamics and control. Oxford University Press, Oxford

Charnov EI (1976) Optimal foraging: the marginal value theorem. Theor Popul Biol 9:129-136

Chatterjee S, Bandyopadhyay M, Chattopadhyay J (2006) Proper predation makes the system disease free-conclusion drawn from an eco-epidemiological model. J Biol Syst 14(4):599-616
Chatterjee S, Kundu K, Chattopadhyay J (2007) Role of horizontal incidence in the occurrence and control of chaos in an ecoepidemiological system. Math Med Biol 24:301-326

Chattopadhyay J, Arino O (1999) A predator-prey model with disease in the prey. Nonlinear Anal 36:747-766

Das KP, Chatterjee S, Chattopadhyay J (2009) Disease in prey population and body size of intermediate predator reduce the prevalence of chaos-conclusion drawn from Hastings-Powell model. Ecol Complex 6(3):363-374

Das KP, Chatterjee S, Chattopadhyay J (2010) Occurrence of chaos and its possible control in a predator-prey model with density dependent disease-induced mortality on predator population. J Biol Syst 18(2):399-435

Diekmann O, Heesterbeek JAP, Metz JAJ (1990) On the definition and the computation of the basic reproductive ratio $R_{0}$ in models for infectious diseases in heterogeneous populations. J Math Biol 28:365-382

Dobson A, Hudson PJ (1986) Parasites, disease and the structure of ecological communities. Trends Ecol Evol 1:11-15

Dobson AP (1988) The population biology of parasite induced changes in host behaviour. Q Rev Biol 63:139-165

Fenton A, Rands SA (2006) The impact of parasite manipulation and predator foraging behavior on predator prey communities. Ecology 87:2832-2841

Freedman HI (1990) A model of predator-prey dynamics modified by the action of parasite. Math Biosci 99:143-155

Fryxell JM, Lundberg P (1994) Diet choice and predator-prey dynamics. Evol Ecol 8:407-421

Gilpin ME (1979) Spiral chaos in a predator-prey model. Am Nat 107:306-308

Han L, Ma Z, Hethcote HW (2001) Four predator prey models with infectious diseases. Math Comput Model 34:849-858

Haque M, Venturino E (2006) The role of transmissible diseases in the Holling-Tanner predator-prey model. Theor Popul Biol 70:273-288

Hastings A, Powell T (1991) Chaos in three-species food chain. Ecology 72(3):896-903

Hadeler KP, Freedman HI (1989) Predator-prey populations with parasitic infection. J Math Biol 27:609-631

Hethcote HW, Han WWL, Ma Zhien (2004) A predator-prey model with infected prey. Theor Popul Biol 66:259-268

Hsieh YH, Hsiao CK (2008) Predator-prey model with disease infection in both populations. Math Med Biol 25(3):247-266

Hilker FM, Schmitz K (2008) Disease-induced stabilization of predator-prey oscillations. J Theor Biol 255:299-306

Hutson V, Law R (1985) Permanent coexistence in general models of three interacting species. J Math Biol 21:289-298

Hofbauer J (1986) Saturated equilibria, permanence and stability for ecological systems. In: Groos L, Hallam $\mathrm{T}$, Levin $\mathrm{S}$ (eds) Mathematical Ecology, Proc. Trieste. World Scientific, Singapore

Holt RD, Dobson AP, Begon M, Bowers RG, Schauber EM (2003) Parasite establishment inhost communities. Ecol Lett 6:837-842

Kermack WO, McKendrick AG (1927) Contributions to the mathematical theory of epidemics, part 1 . Proc R Soc Lond Ser A 115:700-721

Lotka AJ (1925) Relation between birth rates and death rates. Science 26:21-22

Mandal AK, Kundu K, Chatterjee P, Chattopadhyay J (2009) An ecoepidemiological study with Parasitic attack and alternative prey. J Biol Syst 17(2):269-282

Murdoch WW (1969) Switching in general predators: experiments on predator specificity and stability of prey populations. Ecol Monogr 39:335-354

Oslen LF, Truty GL, Schaffer WM (1988) Oscillation and chaos in epidemics: a non-linear dynamic study of six childhood diseases in Copenhagen, Denmark. Theor Popul Biol 33:344-370 
Pielou EC (1969) Introduction to Mathematical Ecology. Wiley, New York

Rai V, Sreenivasan R (1993) Period-doubling bifurcations leading to chaos in a model food chain. Ecol Model 69(1-2):63-77

Ruxton GD (1994) Low levels of immigration between chaotic populations can reduce system extinctions by inducing asynchronous cycles Proc. R Soc Lond B 256:189-193

Rosenzweig ML, MacArthur RH (1963) Graphical representation and stability conditions of predator-prey interactions. Am Nat 97:209-223

Schaffer WM, Kot M (1985a) Nearly one dimensional dynamics in an epidemic. J Theor Biol 112:403-427

Schaffer WM, Kot M (1985b) Do strange attractors govern ecological systems? BioScience 35:342-350

Schaffer WM, Kot M (1986a) Chaos in ecological systems: the coals that Newcastle forgot. Trends Ecol Evol 1:58-63

Schaffer WM, Kot M (1986b) Differential systems in ecology and epidemiology. In: Holden AV (ed). Chaos: an introduction. University of Manchester Press, Manchester, pp 158-178

Sait SM, Liu WC, Thompson DJ, Godfrey HCJ, Begon M (2000) Invasion sequence affects predator-prey dynamics in a multispecies interaction. Nature 405:448-450
Stiefs D, Venturino E, Feude U (2009) Evidence of chaos in ecoepidemic models. Math Biosci Eng 6:855-871

Upadhyay R, Bairagi N, Kundu K, Chattopadhyay J (2007) Chaos in eco-epidemiological problem of Salton Sea and its possible control. Appl Math Comput 196:392-401

Van Baalen M, Krivan V, Van Rijn PCJ, Sabelis MW (2001) Alternative food, switching predators, and the persistence of predator-prey systems. Am Nat 157:512-524

Venturino E (2001) The effects of diseases on competing species. Math Biosci 174(2):111-131

Venturino E (2002) Epidemics in predator-prey model: disease in the predators. IMA J Math Appl Med Biol 19:185-205

Venturino E (1994) The influence of disease on Lotka-Volterra systems. Rky Mt J Math 24:381-402

Venturino E (1995) Epidemics in predator-prey models: disease in the prey. In: Arino O, Axelrod D, Kimmel M, Langlais M (eds) Mathematical population dynamics: analysis of heterogeneity, vol 1. Theory of EpidemicsWuerz Publishing, Winnipeg, pp 381-393

Xiao Y, Chen L (2001) Modelling and analysis of a predator-prey model with disease in the prey. Math Biosci 171:59-82 